\title{
Target-induced 3D DNA Network Structure as a Novel Signal Amplifier for Ultra-Sensitive Electrochemiluminescence Detection of MicroRNAs
}

Yue Zhang, Yaqin Chai, Haijun Wang, * and Ruo Yuan*

Key Laboratory of Luminescence and Real-Time Analytical Chemistry (Southwest

University), Ministry of Education, College of Chemistry and Chemical Engineering,

Southwest University, Chongqing 400715, People's Republic of China 


\section{Table of Contents}

Reagents and Materials ---- S3

Instruments ---- S4

Preparation of MBs-H1 ---- S5

Preparation of H2-AuNPs-Output DNA ---- S5

Preparation of DNA Duplex Probes (S1-ABEI/S2-DA) ---- S6

Fluorescence Spectral Characterization of BNQDs ---- S7

ECL Behaviors Comparison of Different Luminous Nanocomposites ---- S7

PAGE Analysis ---- S8

Study of the Double Quenching Effect of DA---- S10

Optimization of the ECL Biosensor ---- S11

Calculation of LOD and LOQ ---- S12

References ---- S13 


\section{Reagents and Materials.}

Gold chloride tetrahydrate $\left(\mathrm{HAuCl}_{4} \cdot 4 \mathrm{H}_{2} \mathrm{O}\right), 1$-Ethyl-3- [3- (dimethylamino)-propyl] carbodiimide hydrochloride (EDC), N-hydroxy succinimide (NHS), Hexanethiol (HT) (96\%) and tris (2-carboxyethyl) phosphine hydrochloride (TCEP) were all purchased from Sigma-Aldrich Co. (St. Louis, MO, U.S.A.). N- (4-aminobutyl)-Nethylisoluminol (ABEI), dopamine (DA), $\mathrm{Ru}(\text { bpy })_{3} \mathrm{Cl}_{2} \cdot 6 \mathrm{H}_{2} \mathrm{O}$, Nafion $(5 \%, w t / w t)$, $\mathrm{H}_{2} \mathrm{Pt}_{2} \mathrm{Cl}_{6} \cdot 6 \mathrm{H}_{2} \mathrm{O}$ and 3-thiophenemalonic acid (TA) were acquired from Suna Tech Inc. (Suzhou, China). Magnetic beads (MBs) and concentrated ammonia were obtained from Tianjin BaseLine ChromTech Research Centre (Tianjin, China) and Titan Technology Co., Ltd. (Shanghai, China) respectively. Iodine and potassium iodide were got from Teng Zhun Biological Co., Ltd (Shanghai, China) and Meryer Chemical Technology Co. Ltd. (Shanghai, China) respectively. Exonuclease III (Exo III) was provided by Dingguo Biotechnology Co., Ltd. (Chongqing, China). Boric acid and the oligonucleotides (see Table S1) used in this work were synthesized and refined by Sangon Inc. (Shanghai, China). Human breast cancer cells (MCF-7) and cervical cancer cells (Hela) were purchased from the Type Culture Collection of the Chinese Academy of Sciences (Shanghai, China). Meanwhile, the involved buffers were outlined as follows. DNA reserve buffer $(\mathrm{pH}=8.0)$ : $1 \times \mathrm{TE}$ buffer $(10 \mathrm{mM}$ Tris-HCl, $1.0 \mathrm{mM}$ EDTA). DNA annealing buffer and hybridization buffer (10 mM Tris-HCl, $1 \mathrm{mM}$ EDTA, $15 \mathrm{mM} \mathrm{MgCl}_{2}, 200 \mathrm{mM} \mathrm{NaCl}, \mathrm{pH}=8.0$ ). The detection phosphate buffer (PBS, 
$\mathrm{pH}=7.4): 0.1 \mathrm{M} \mathrm{Na}_{2} \mathrm{HPO}_{4}, 0.1 \mathrm{M} \mathrm{KH}_{2} \mathrm{PO}_{4}$, and $0.1 \mathrm{M} \mathrm{KCl}$. Deionized water was used throughout the experiment. All reagents were analytical grade and solutions were prepared using ultrapure water (specific resistance of $18 \mathrm{M} \Omega \cdot \mathrm{cm}$ ).

Table S1. All DNA and miRNA Sequences Used in the Experiment

Name

Sequence $\left(5^{\prime}\right.$ to $\left.3^{\prime}\right)$

MicroRNA-21

UAG CUU AUC AGA CUG AUG UUG A

MicroRNA-155

UUA AUG CUA AUC GUG AUA GGG GU

MicroRNA-141

UAA CAC UGU CUG GUA AAG AUG G

MicroRNA-let-7a

UGA GGU AGU AGG UUG UAU AGU U

$\mathrm{H} 1$

$\mathrm{NH}_{2}$-TCAACATCAGTCTGATAAGCTACC

ATGTCCATGTGTAGATAGCTTATCAGACT

$\mathrm{H} 2$

SH-TGATAAGCTATCTACACATGGACATGG

TAGCTTATCAGACTCCATGTCCATGTGTAGA

S1 COOH-ACCCTCTGTAGGTTGTATAGAAAACAGAGGGT-SH

$\mathrm{S} 2$

COOH-TTTTCTATACAACCTACTACCTCA

Output DNA

SH-TGAGGTAGTAGGTTGTATAGTT

\section{Instruments.}

The ECL measurement was conducted with a model MPI-A ECL analyzer (Xi'an Remax Electronic Science \& Technology Co. Ltd., Xi'an, China). Cyclic voltammetry (CV) and electrochemical impedance spectroscopy (EIS) measurements were conducted with a CHI 660E electrochemical workstation $(\mathrm{CH}$ Instruments Inc., 
Shanghai, China). The F7000 fluorescence spectrophotometer (Hitachi HighTechScience Co., Tokyo, Japan) was used for photoluminescence property study. The visual photos were acquired by UV transilluminator (Clinx Science Instruments Co., Ltd). Furthermore, the transmission electron microscope (TEM, H600, Hitachi Instrument, Japan), high-resolution TEM (HRTEM) and atomic force microscopy (AFM, Bruker Co. Germany) were applied for material characterization. Polyacrylamide gel electrophoresis (PAGE) were carried out on the DYCP-31E electrophoresis apparatus (WoDeLife Sciences Instrument Co., Ltd., China). In the whole experiment course, the three-electrode strategy which was made up of a modified glassy carbon electrode (GCE, $\Phi=4 \mathrm{~mm}$ ) as the working electrode, an $\mathrm{Ag} / \mathrm{AgCl}$ electrode (sat. $\mathrm{KCl}$ ) as the reference electrode, and a platinum wire as the auxiliary electrode was very necessary to accomplish the experimental detection.

\section{Preparation of MBs-H1.}

The hairpin DNA H1 was heated to $95{ }^{\circ} \mathrm{C}$ for $5 \mathrm{~min}$ and then slowly cooled to room temperature for $1 \mathrm{~h}$. MBs-H1 were prepared by the following procedures using NHS and EDC as the cross-linking agent. Firstly, $\mathrm{COOH}$-modified MBs were activated for $2 \mathrm{~h}$ by $0.05 \mathrm{M}$ NHS and $0.2 \mathrm{M}$ EDC. Then, $5 \mu \mathrm{M}$ H1 which had been annealed was added to the above mixture solution, and reacted at $4{ }^{\circ} \mathrm{C}$ overnight with stirring for the complete conjugation of $\mathrm{H} 1$ on MBs. Finally, MBs-H1 were magnetically separated and re-dispersed in $1 \mathrm{~mL}$ DNA reserve buffer and stored at $4{ }^{\circ} \mathrm{C}$ for later use. 


\section{Preparation of H2-AuNPs-Output DNA.}

A typical method for synthesizing AuNPs was to add $0.6 \mathrm{~mL}$ of $0.1 \mathrm{M}$ ice-cold $\mathrm{NaBH}_{4}$ solution into $20 \mathrm{~mL}$ of $0.25 \mathrm{mM} \mathrm{HAuCl}_{4}$ solution with stirring until the color of the mixed solution changed to orange-red. After the mixed solution was cooled at room temperature, it was centrifuged to obtain AuNPs. Before use, H2 was annealed at $95{ }^{\circ} \mathrm{C}$ for $5 \mathrm{~min}$ and then cooled down to room temperature slowly to form a hairpin structure. In order to prevent the formation of dimers of $\mathrm{H} 2$ and output DNA, $100 \mu \mathrm{L}$ of $5 \mu \mathrm{M} \mathrm{H} 2,100 \mu \mathrm{L}$ of $50 \mu \mathrm{M}$ output DNA (molar ratio of the H2: output DNA $=1: 10$ ), and $20 \mu \mathrm{L}$ of $10 \mathrm{mM}$ TCEP were mixed, and then incubated at room temperature for 2 $\mathrm{h}$ to reduce the disulfide bond. After that, $500 \mu \mathrm{L}$ of AuNPs were slowly added to the above solution and stirred for $16 \mathrm{~h}$. Finally, the obtained H2-AuNPs-output DNA was centrifuged and re-dispersed three times to store at $4{ }^{\circ} \mathrm{C}$ for later use.

\section{Preparation of DNA Duplex Probes (S1-ABEI/S2-DA).}

The procedure for preparing DNA duplex probes was as follows. At first, $200 \mu \mathrm{L}$ of $5 \mu \mathrm{M}$ signal probes (S1), $20 \mu \mathrm{L}$ of $10 \mathrm{mM}$ TCEP and $200 \mu \mathrm{L}$ of $8 \mu \mathrm{M}$ toehold probes (S2) were activated by the cross-linking agent EDC (0.2 M) and NHS (0.05 M) for $2 \mathrm{~h}$, respectively. Then, $0.02 \mathrm{M}$ of ABEI solution and $0.02 \mathrm{M}$ of DA solution were added into the above solution respectively, which were reacted overnight at $4{ }^{\circ} \mathrm{C}$ with stirring. Finally, the prepared S1-ABEI and S2-DA were mixed and hybridized at $37^{\circ} \mathrm{C}$ for $2 \mathrm{~h}$. Hence, the DNA duplex probes were synthesized successfully and stored at $4{ }^{\circ} \mathrm{C}$ for later use. 


\section{Fluorescence Spectral Characterization of BNQDs}
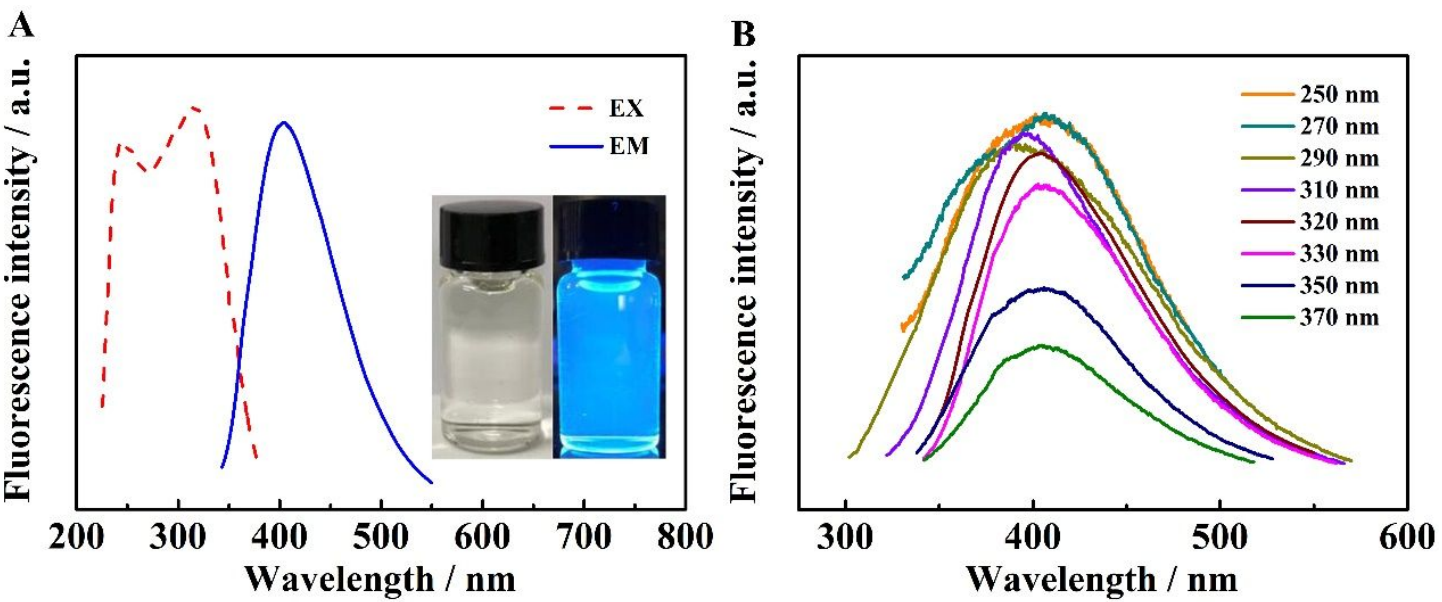

Figure S1. (A) Excitation (red dashed line) and emission (blue solid line) spectra of BNQDs. Inset: Photograph of BNQDs solution under UV light (right) and visible light (left). (B) Fluorescence emission spectrum of BNQDs at different excitation wavelengths.

\section{ECL Behaviors Comparison of Different Luminous Nanocomposites.}

In order to obtain a stable and strong initial signal, we compared the ECL behaviors of three different nanocomposites. When Ru/PtNPs dispersed in the nafion solution (Ru/PtNPs/Nafion) was dropped on the clean GCE surface, it could be seen from Figure S2 that only a small ECL signal (326.3 a.u.) was obtained by performing the ECL detection without the co-reactant (curve a). However, the ECL intensity increased to 4865.7 a.u. after the BNQDs were added directly to the detection bottom liquid (0.1 M PBS, $\mathrm{pH}=7.4$ ), because BNQDs as a co-reactant could significantly enhance the ECL signal of $\mathrm{Ru}(\mathrm{bpy})_{3}{ }^{2+}$ (curve b). Most importantly, when the co-reactant BNQDs and $\mathrm{Ru} / \mathrm{PtNPs} / \mathrm{Nafion}$ were prepared as nanocomposite (BNQDs/Ru/PtNPs/Nafion) for 
GCE modification, the ECL intensity was further increased to 10303.9 a.u. due to the shorter electron-transfer path and less energy loss (curve c).

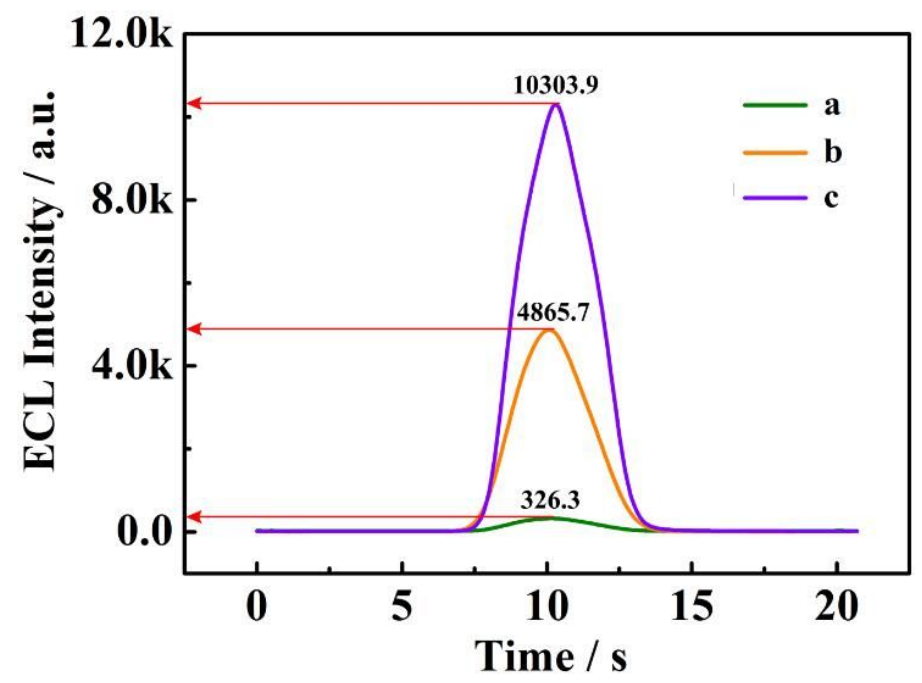

Figure S2. The ECL behaviors of electrode modified with (a) Ru/PtNPs/Nafion, (b) $\mathrm{Ru} / \mathrm{PtNPs} /$ Nafion with BNQDs in the detection bottom liquid, (c) $\mathrm{BNQDs} / \mathrm{Ru} / \mathrm{PtNPs} / \mathrm{Nafion}$. All the ECL responses were tested in PBS (0.1 M, $\mathrm{pH}=7.4)$. The voltage of photomultiplier tube (PTM) was set at $800 \mathrm{~V}$ and the potential scan was from 0.2 to $1.25 \mathrm{~V}$ at a scanning rate of $100 \mathrm{mV} \mathrm{s}^{-1}$.

\section{PAGE Analysis.}

The $16 \%$ PAGE analysis was carried out to corroborate the feasibility of the strategy and the interactions among these DNA sequences. Figure S3A showed the reaction of Process A of this strategy. Three clear bands were observed for H1, H2 and output DNA, respectively (lanes 1, 2 and 3). The mixture of the two metastable DNA hairpins did not show any new band (lane 4), evidencing a low background of the catalyzed hairpin assembly (CHA) reaction. When miRNA-21 was added to the two hairpins for incubation, a new band (lane 5) appeared, indicating the formation of the product H1- 
$\mathrm{H} 2$ of CHA. output DNA was added to lane 4 and lane 5, respectively, showing that there was only one more band, while the other results did not change (lane 6 and lane 7), demonstrating that output DNA had no effect on the CHA reaction. For Process B, Figure S3B demonstrated its feasibility. The three clear bands were output DNA, S1 and S2, respectively (lanes 1, 2 and 3). The DNA mixture containing output DNA, S1 and S2 was subjected to a hybridization reaction at $37^{\circ} \mathrm{C}$ for $2 \mathrm{~h}$, and two bands of the output DNA-S2 complex and S1 were observed (lane 4). Exo III (2 U/ $\mu \mathrm{L})$ was added to the hybridized DNA mixture and reacted at $37^{\circ} \mathrm{C}$ for $30 \mathrm{~min}$, followed by inactivation of this exonuclease at $85^{\circ} \mathrm{C}$ for $15 \mathrm{~min}$. Two bands of $\mathrm{S} 1$ and output DNA could be observed (lane 5), and it could be shown that S2 had been completely cleaved by Exo III. The above results confirmed the interactions among these DNA sequences.

A

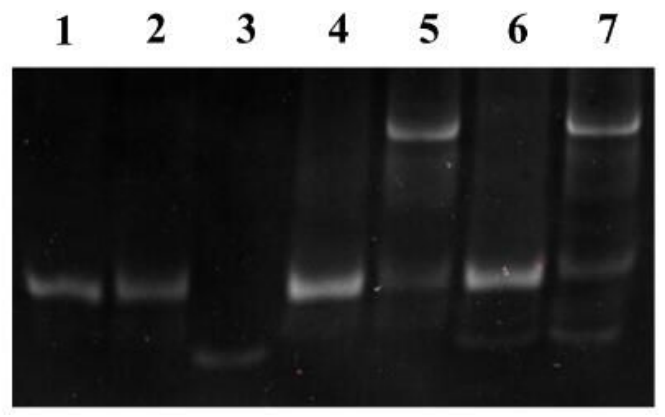

B

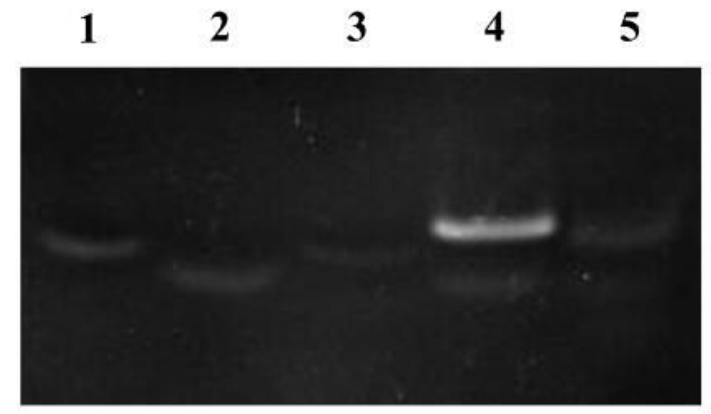

Figure S3. Characterization of the interaction between DNA sequences by PAGE. (A)

Lane 1: H1; Lane 2: H2; Lane 3: output DNA; Lane 4: H1+H2; Lane 5: H1+H2+miRNA-21; Lane 6: H1+H2+output DNA; Lane 7: $\mathrm{H} 1+\mathrm{H} 2+$ miRNA21+output DNA. (B) Lane 1: output DNA; Lane 2: S1; Lane 3: S2; Lane 4: output DNA+S1+S2; Lane 5: output DNA+S1+S2+ Exo III (2 U/ $\mu \mathrm{L})$. 


\section{Study of the Double Quenching Effect of DA.}

In order to verify the double quenching effect of DA to the ECL of ABEI and $\mathrm{Ru}(\mathrm{bpy}) 3_{3}{ }^{2+}$, some supplementary experiments were also performed. At first, it has been reported that dopamine (DA) could quench the ECL signal of ABEI. ${ }^{1,2}$ For further proving that, the ECL signals of the ABEI solution without and with DA were recorded. As seen from Figure S4A, a high initial ECL intensity was obtained (curve a) in the ABEI solution, while about 2892.7 a.u. decline of the ECL intensity was acquired when DA was added. In addition, it has also been proven that DA was a classic quencher to the ECL of Ru(bpy) $3_{3}{ }^{2+} .1,3$ Similarly, a comparative experiment was also performed in $\mathrm{Ru}(\mathrm{bpy}) 3_{3}{ }^{2+}$ solution. As shown in Figure $\mathrm{S} 4 \mathrm{~B}$, about 4386.9 a.u. decline of the ECL intensity was acquired after the addition of $\mathrm{DA}$ to $\mathrm{Ru}(\mathrm{bpy})_{3^{2+}}$ solution. The experimental results above demonstrated that DA had double quenching effect to the ECL of ABEI and Ru(bpy $)_{3}{ }^{2+}$ in this work.

A

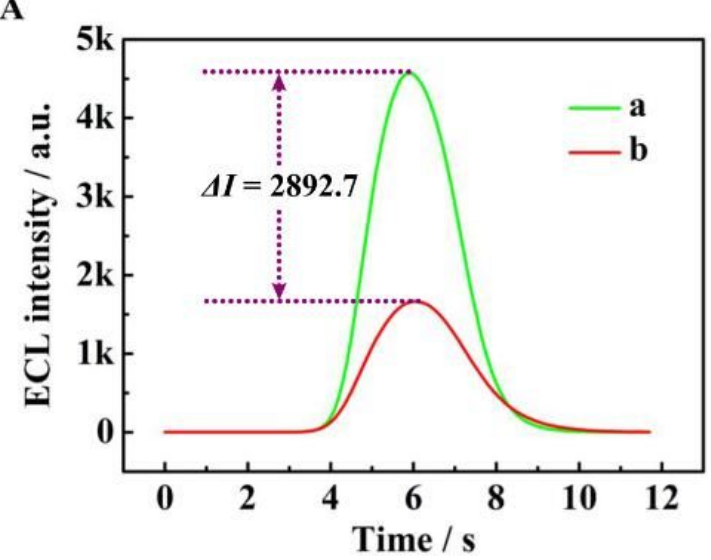

B

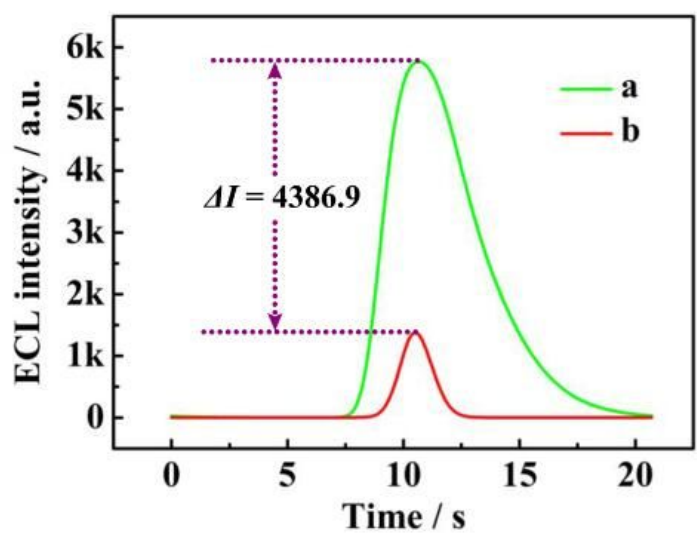

Figure S4. (A) ECL intensities of GCE in ABEI solution (a) and that in ABEI solution with the presence of DA (b). (B) ECL intensities of GCE in Ru(bpy) $3^{2+}$ solution (a) and that in $\mathrm{Ru}(\mathrm{bpy}) 3^{2+}$ solution with the presence of DA (b). 


\section{Optimization of the ECL Biosensor.}

In order to achieve the optimal performance of the biosensor, we focused on the optimal conditions of two key parameters. One was the mass ratio of iodine to potassium iodide used to dissolve AuNPs, and another was the cleaving time of Exo III. It could be seen from Figure S5A that the value of $m\left(\mathrm{I}_{2}\right) / m\left(\mathrm{I}^{-}\right)$affected the dissolution effect of AuNPs. When the ratio was too small, there was not enough $\mathrm{I}_{3}$ in the solution, which affected the ability of oxidizing to weaken the ability to dissolve AuNPs. When the ratio was too large, $\mathrm{I}^{-}$in the solution reacted with $\mathrm{I}_{2}$ to form $\mathrm{I}_{3}{ }^{-}$, and the decrease in the amount of $\mathrm{I}^{-}$resulted in insufficient coordination ability with AuNPs to affect the dissolution of the AuNPs. Therefore, the value of $m\left(\mathrm{I}_{2}\right) / m\left(\mathrm{I}^{-}\right)$had a better effect of dissolving AuNPs within a certain range (1:4 to 3:10), and 1:4 (0.2 $\mathrm{g} / \mathrm{ml} \mathrm{KI}$ ) was selected as a suitable mass ratio. Figure S5B showed the effect of Exo III cleaving time on the biosensor. When the Exo III cleaving time was increased from 0 to $70 \mathrm{~min}$, the ECL response increased accordingly and reached a plateau at $60 \mathrm{~min}$, indicating that the optimal cleaving time was achieved at $60 \mathrm{~min}$.
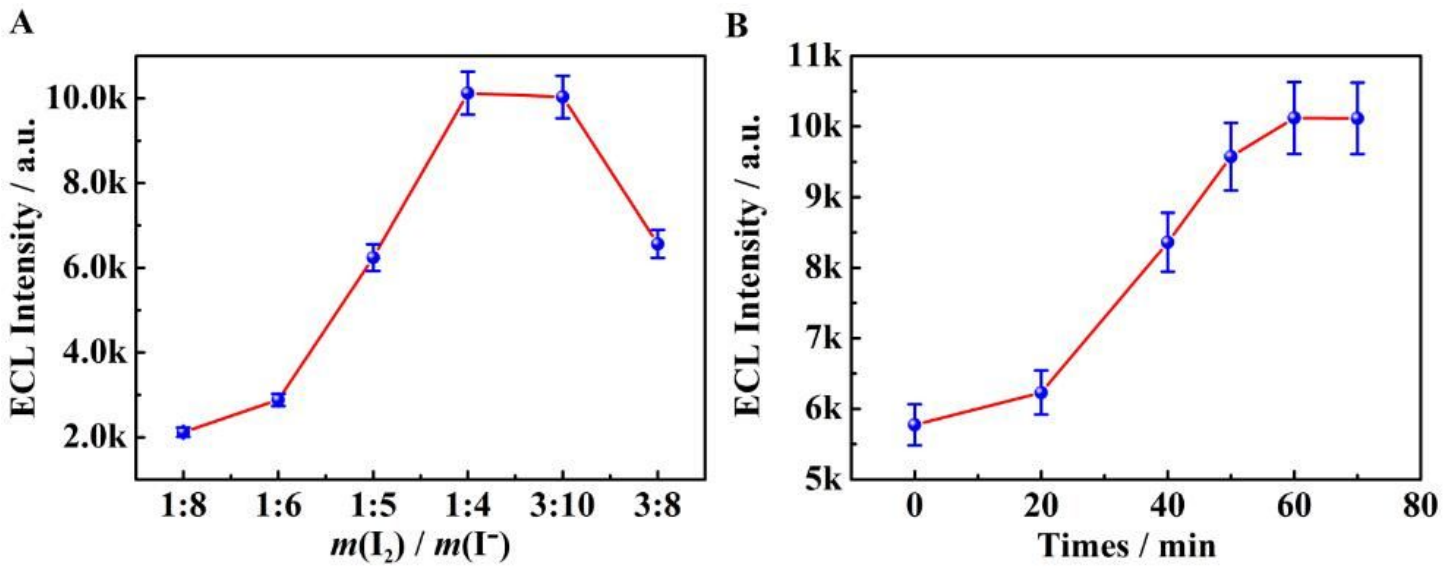

Figure S5. Influence of (A) the value of $m\left(\mathrm{I}_{2}\right) / m\left(\mathrm{I}^{-}\right)$and (B) Exo III cleaving time on 
ECL response.

\section{Calculation of LOD and LOQ.}

According to the related literatures and the IUPAC recommendation in $1976,{ }^{4-10}$ the limit of detection (LOD) and the limit of quantitation (LOQ) in our work was defined as $\mathrm{LOD}=3 S_{\mathrm{B}} / m$ and LOQ $=10 S_{\mathrm{B}} / m$, respectively, where $S_{B}$ was the standard deviation of the blank signals $\left(n_{\mathrm{B}}=20\right)$ and $m$ was the analytical sensitivity which could be estimated as the slope of calibration curve at lower concentration ranges. And we plotted the calibration curve showing ECL intensity values with the change of targets at lower concentration ranges shown in Figure S6. As shown in Figure S6, the insert presented that ECL intensity values ( $I$ / a.u.) was linearly related to the concentration of miRNA-21 $(c / \mathrm{fM})$ at a low concentration range. The corresponding linear equation was $I=246861.56 c+1271.66$ and the $S_{\mathrm{B}}$ of twenty times zero-dose was about 27.15. Therefore, the LOD and LOQ of the proposed ECL biosensor were $0.33 \mathrm{aM}$ and 1.0 $\mathrm{aM}(\mathrm{LOD}=3 \times 27.15 \div 246861.56=0.00033 \mathrm{fM}=0.33 \mathrm{aM}, \mathrm{LOQ}=10 \times 27.15 \div$ $246861.56=0.001 \mathrm{fM}=1 \mathrm{aM})$, respectively. 


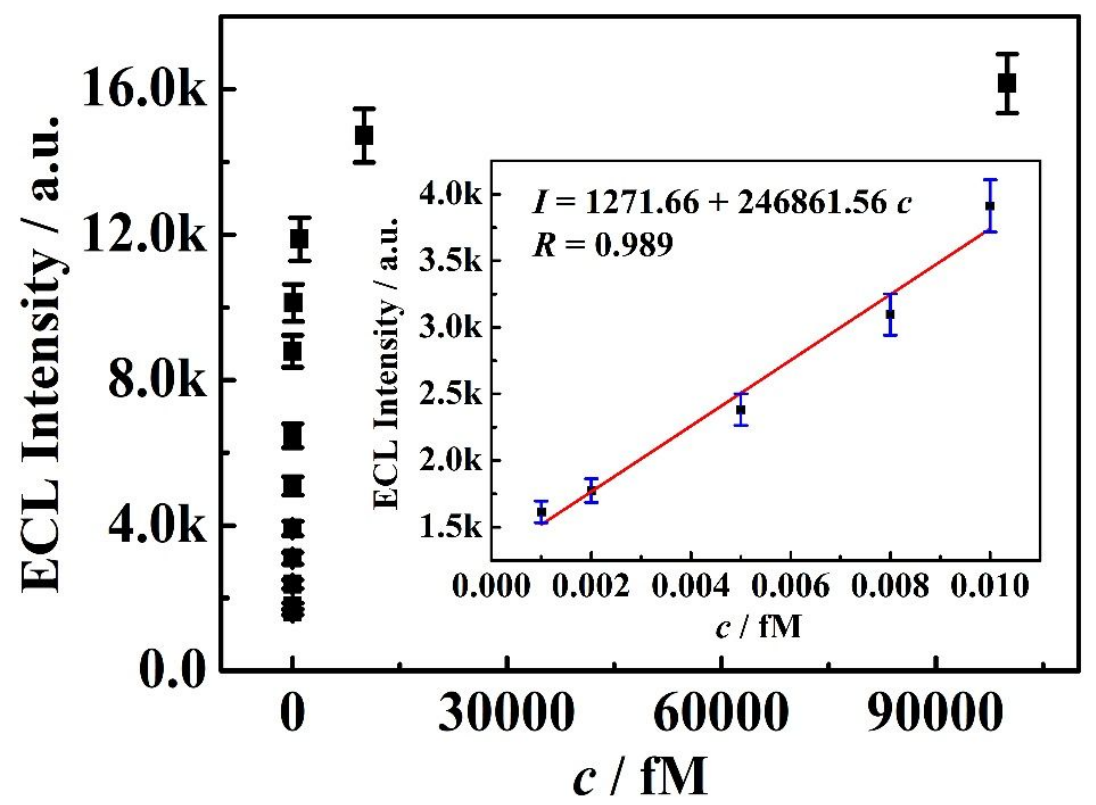

Figure S6. The changed trends of ECL responses with the concentrations of miRNA21. The insert showed the calibration curve of ECL responses with the change of target at lower concentration ranges.

\section{References}

1. Wang, H. J.; Peng, L. Y.; Chai, Y. Q.; Yuan, R. Anal. Chem. 2017, 89, 11076-11082.

2. Zhang, P.; Wu, X. Y.; Yuan, R.; Chai, Y. Q. Anal. Chem. 2015, 87, 3202-3207.

3. Li, Q.; Zheng, J.Y.; Yan, Y. L.; Zhao, Y. S.; Yao, J. N. Adv. Mater. 2012, 24, 47454749.

4. Buck, R. P.; Lindner, E. Pure Appl. Chem. 1994, 66, 2527-2536.

5. Currie, L. A. Pure Appl. Chem. 1995, 67, 1699-1699.

6. Radi, A. E.; Acero Sánchez, J. L.; Baldrich, E.; O'Sullivan, C. K. J. Am. Chem. Soc. 2006, 128, 117-124.

7. Akter, R.; Rahman, M. A.; Rhee, C. K. Anal. Chem. 2012, 84, 6407-6415.

8. IUPAC Pure Appl. Chem. 1976, 45, 107. 
9. Long, G.L.; Winefordner, J.D. Anal. Chem. 1983, 55, 712A-724A.

10. Shrivastava, A.; Gupta, V. B. Chronicles of Young Scientists 2011, $2,21$. 